\title{
Giant adrenal germ cell tumour in a 59-year-old woman
}

\author{
Lei Chen, MD; Lu Fang, MM; Zhiqi Liu, MM; Dexin Yu, MD; Daming Wang, MM; Yi Wang, MD; \\ Dongdong Xie, MD; Jie Min, MD; Demao Ding, MD; Tao Zhang, MD; Ci Zou, MD; Zhiqiang Zhang, MM
}

Department of Urology, The Second Affiliated Hospital of Anhui Medical University, Anhui Province, China

Cite as: Can Urol Assoc J 2016;10(5-6):E201-3. http://dx.doi.org/10.5489/cuaj.2904 Published online May 12, 2016.

\section{Abstract}

Adrenal germ cell tumour is very rare. We report a case of a 59-year-old woman who presented with right flank discomfort. The laboratory examinations were normal and the chest computed tomography (CT) showed right pleural effusion. The abdominal CT scan revealed a large mass on the right adrenal gland. The patient underwent an adrenalectomy. Histopathologic examination and immunohistochemical findings were consistent with mixed germ cell tumour. Three months later following the operation, the patient was admitted to our hospital again with chest tightness and shortness of breath. The chest CT showed right pleural effusion recurrence and enlargement of mediastinal lymph nodes and right hilar lymph nodes. The patient had right supraclavicular lymphadenectasis on physical examination. Fine needle aspiration cytology from the supraclavicular lymph nodes showed groups of malignant tumour cells. The patient died within 6 months postoperatively. In this case, the lymph node pathway played an important role in the metastatic procedure.

\section{Introduction}

Extragonadal germ cell tumours (GCTs) are uncommon and represent $2 \%$ to $5 \%$ of adult germ cell malignancies. ${ }^{1,2}$ The histogenesis of extragonadal GCT is unknown. We present an extremely rare case of an adrenal mixed GCT composed of dysgerminoma and embryonal carcinoma. To our knowledge, this is the first report of GCT in the adrenal gland.

\section{Case report}

A 59-year-old woman was admitted to hospital with a fourmonth history of right flank discomfort. Physical examinations and laboratory findings were normal. An abdominal computed tomography (CT) scan revealed a large, heterogeneous, poorly defined solid mass measuring $7.6 \times 6.7 \times 9.0 \mathrm{~cm}$ on the right adrenal gland (Fig. 1A). The chest CT showed right pleural effusion. Moreover, the thoracic drainage device was closed. There were no tumour cells in pleural effusion. A successful right adrenalectomy was thus performed on the patient. Observed by the naked eye, we partially circumscribed and encapsulated the excised tumour with maximum measurement of $9.0 \mathrm{~cm}$. On the cut section, it had a uniform, yellowish, solid and partially nodular appearance within the cystic necrosis area.

Histologically, the tumour consisted of diffuse sheets, alveolar aggregates, and cords of incohesive round monotonous cells. These cells contained moderate amounts of eosinophilic granular cytoplasm and eccentrically located large round nuclei with prominent nucleoli. Cords of tumour cells were separated by fine connective tissue septa containing many lymphocytes (Fig. 2A). On immunohistochemical staining, the tumour cells were positive for human placental lactogen (Fig. 2B), c-kit, Vim, CEA, CgA, S-100, CD117 and alpha-fetoprotein (weakly positive), but negative for EMA, Syn, CD20, CD30, placental alkaline phosphatase, CK7, $\mathrm{CK} 2$, and a-inhibin. These results confirmed a final diagnosis of mixed GCT with dysgerminoma and embryonal carcinoma.

Initially, the tumour was considered metastatic. Postoperative ultrasonography, pelvic CT scan, however, showed no abnormal findings in the bilateral ovaries. The patient's serum concentrations of the tumour markers, such as alpha-fetoprotein (16 ug/L), CA-125 and carcinoembryonic antigen, were within normal ranges. Chemotherapy was recommended, but the patient refused it.

Three months after the operation, the patient presented to the respiratory department with a three-day history of chest tightness and shortness of breath. The computed tomography scan revealed a large amount of pleural effusion on the right side and enlargement of mediastinal lymph nodes and right hilar lymph nodes (Fig.1B). On physical examination she had right supraclavicular lymphadenectasis. Subsequently, 


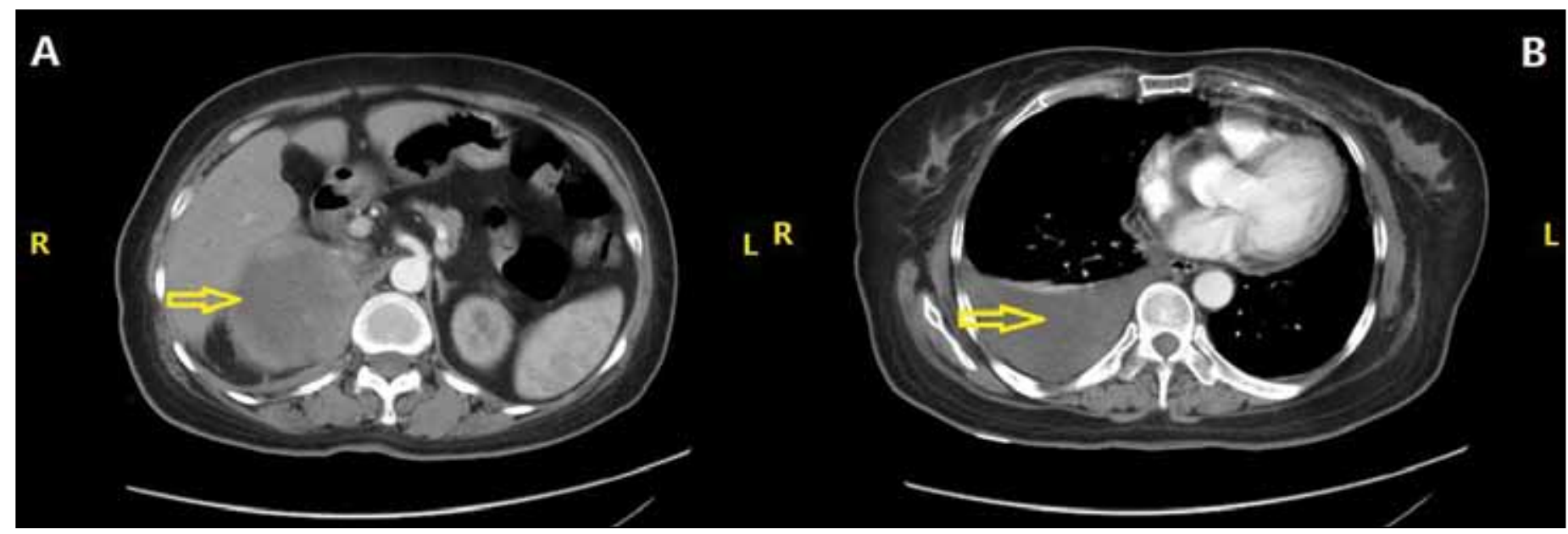

Fig. 1. Contrast-enhanced abdomen CT scan shows bulky adrenal mass with large central unenhancing necrotic region, peripheral heterogeneously enhancing solid component (A); and vomputed tomography scan of the chest showed right pleural effusion (B).

we assessed the closed thoracic drainage and performed a superficial fine needle aspiration biopsy. Tumour cells still had not been found in pleural effusion. However, fine needle aspiration cytology from the supraclavicular lymph nodes showed groups of malignant tumour cells (Fig. 2C). Adjuvant chemotherapy and fibreoptic bronchoscopy were recommended, both of which the patient had declined. Ultimately, the patient died within six months postoperatively.

\section{Discussion}

Extragonadal GCT was first described in 1939. Its etiology is unknown and whether extragonadal GCTs are metastatic remains controversial. There are two theories to explain this phenomenon. The first is spontaneous regression of the primary GCT after its metastasis. Possible mechanisms are an immune response or ischemia caused by the disseminated neoplasm due to its high metabolic rate. The second is the de novo development of a primary GCT in extragonadal tissues. ${ }^{3}$ Extragonadal GCTs share the gain of isochromosome $12 \mathrm{p}$ with gonadal GCTs. ${ }^{4}$ In rare cases, extragonadal GCTs have been associated with Klinefelter syndrome. ${ }^{5,6}$ Their anatomic distribution varies widely and includes the mediastinum, ${ }^{7}$ sacrococcygeal region, ${ }^{8}$ neck, retrobulbar, ${ }^{9}$ retroperitoneum, ${ }^{10}$ and other rare sites. ${ }^{11,12}$ GCT rarely involves the adrenal gland. To our knowledge, this is the first report of adrenal mixed GCT composed of dysgerminoma and embryonal carcinoma.

In contrast to mixed GCT composed of dysgerminoma and embryonal carcinoma, primary teratomas of the adrenal gland have been reported. ${ }^{13-16}$ Our unique case differs from most reports of reported adrenal teratomas. On CT scans, teratoma is frequently shown as a heterogeneous fat dense mass with calcifications. Magnetic resonance T2 weighyed images demonstrate teratoma as a highlighted intensity around the tumour components. In our patients'case, the diagnosis of adrenal GCT was difficult to make based on the imaging studies. There were no definite radiologic features

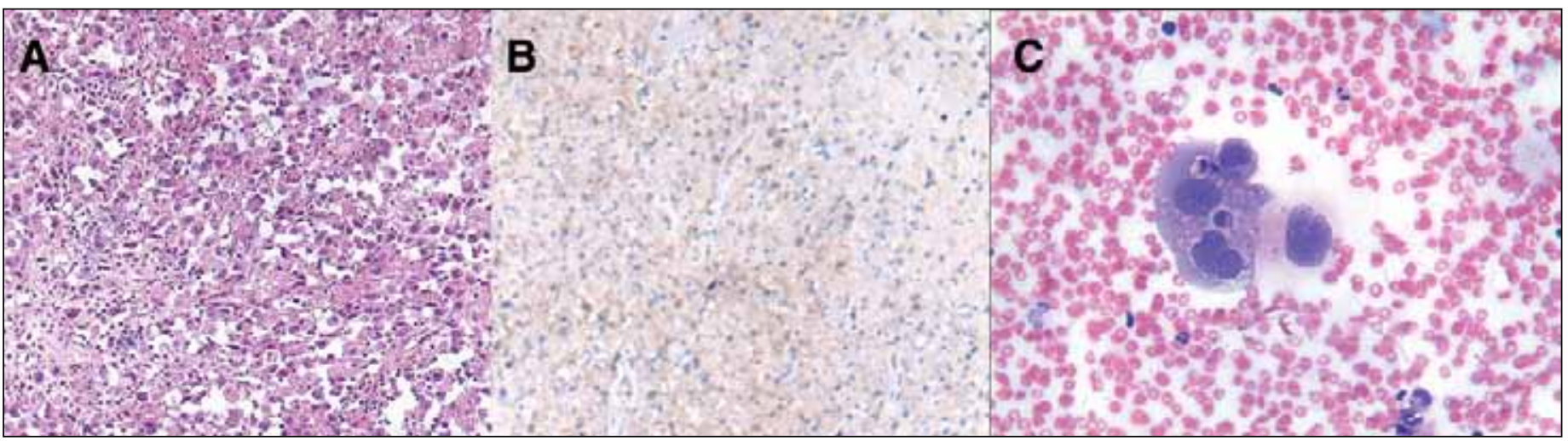

Fig. 2. Microscopic observation of specimens revealed uniform tumor cells with sharply outlined cell membranes, small amounts of cytoplasm and large central nuclei. Tumor cells were typically arranged in nests surrounded by fibrous bands and detached from each other (A), and malignant focus was positive for human chorionic gonadotrophin (B), and fine needle aspiration cytology from the supraclavicular lymph nodes showed groups of malignant tumor cells (C). 
to distinguish primary adrenal GCT from cortisol-producing, adenoma, aldosterone-producing adenoma, adrenal cortical carcinoma, and non-functioning adenoma.

Lymphatic vessels and lymph nodes may not only act as a route of spread, but also sanctuaries of metastatic deposits. ${ }^{17}$ Mixed GCTs are very aggressive and have early metastatic potential to the lymph nodes. It had been reported that lymph nodes were the most common site of metastasis in malignant mixed GCTs. ${ }^{18}$ In this case, the lymph node pathway plays an important role in the metastatic procedure. The patient had right supraclavicular lymphadenectasis three months after the operation, a chest CT revealed pleural effusion on the right side and enlarged mediastinal lymph nodes and right hilar lymph nodes. Ultimately, she died within six months postoperatively.

Although there is no standard treatment for adrenal mixed GCT, complete surgical excision of the lesions may be the only potentially curative treatment. There is little data with long-term followup; moreover, the therapy and prognosis of adrenal mixed GCTs have not been properly clarified. It has been previously reported that patients with primary extragonadal GCTs respond well to cisplatin-based combination chemotherapy, regardless of the site at presentation. ${ }^{19}$ For this reason, the combination of chemotherapy and surgery is the most appropriate treatment strategy.

\section{Conclusion}

Adrenal GCT is rare. Because of the limited number of cases, the treatment of adrenal germinoma has not been established yet. The combination of chemotherapy and surgery is the most appropriate treatment strategy.

Competing interests: The authors declare no competing financial or personal interests.

This paper has been peer-reviewed.

\section{References}

1. Stang $A$, Trabert $B$, Wentzensen $N$, et al. Gonadal and extragonadal germ cell tumours in the United States, 1973-2007. Int J Androl 2012;35:616-25. http://dx.doi.org/10.1111/i.1365-2605.2011.01245.x

2. Trama A, Mallone $\mathrm{S}$, Nicolai N, et al. Burden of testicular, paratesticular and extragonadal germ cell tumours in Europe. Eur J Cancer 2012;48:159-69. http://dx.doi.org/10.1016/i.ejca.2011.08.020

3. Hainsworth JD, Greco FA. Extragonadal germ cell tumours and unrecognized germ cell tumors. Semin Oncol 1992;19:119-27.

4. Bosl GJ, Dmitrovsky E, Reuter V, et al. i(12p): A specific karyotypic abnormality in germ cell tumours. Proc Am Soc Clin Oncol 1989;8:131.

5. Phowthongkum $P$. The second case of de novo intracranial germinoma association with Klinefelter's syndrome. Surg Neurol 2006;66:332. http://dx.doi.org/10.1016/j.surneu.2006.05.041

6. Aguirre $D$, Nieto $K$, Lazos $M$, et al. Extragonadal germ cell tumours are often associated with Klinefelter syndrome. Hum Pathol 2006;37:477. http://dx.doi.org/10.1016/i.humpath.2006.01.029

7. Dalal U, Jora MS, Dalal AK, et al. Primary germ cell tumour of the mediastinum - presenting as a huge mass. Int J Prev Med 2014;5:230-2.

8. Pawar NP, Mahajan SV, Chaudhari RA, et al. Extragonadal GCT: A rare case report of sacrococcygeal pure yolk sac tumour. Indian J Pathol Microbiol 2013;56:329-31. http://dx.doi.org/10.4103/03774929.120421

9. Deb M, Mohanty S, Ananthamurthy A, et al. Atypical extragonadal germ cell tumours. J Indian Assoc Pediatr Surg 2012;17:9-15. http://dx.doi.org/10.4103/0971-9261.91079

10. Misiak P, Piskorz L, Jabło ski $S$, et al. Isolated retroperitoneal mixed germ cell tumours presenting as acute abdomen - case report. Contemp Oncol (Pozn) 2013;17:94-6. http://dx.doi.org/10.5114/ wo.2013.33782

11. Frazier AL, Hale JP, Rodriguez-Galindo C, et al. Revised risk classification for pediatric extracranial germ cell tumours based on 25 years of clinical trial data from the United Kingdom and United States. J Clin Oncol 2015;33:195-201. http://dx.doi.org/10.1200/JC0.2014.58.3369

12. Kishore M, Monappa V, Rao L, et al. Mixed malignant germ cell tumour of third ventricle with hydrocephalus: A rare case with recurrence. J Clin Diagn Res 2014;8:FD03-5. http://dx.doi.org/10.7860/ $\mathrm{jcdr} / 2014 / 9866.5124$

13. Tang $D D$, Zhang XS, Hao ZY, et al. A giant primary retroperitoneal mature cystic teratoma in right adrenal region in a 39-year-old female. Int J Clin Exp Med 2014;7:1611-3.

14. Ciffci I, Cihan T, Koksal Y, et al. Giant mature adrenal cystic teratoma in an infant. Acta Inform Med 2013;21:140-1. http://dx.doi.org/10.5455/aim.2013.21.140-141

15. Shrestha MK, Lalchan S. Adrenal gland teratoma in a 40-year-old woman. Nepal Med Coll J2010;12:201-2.

16. James J, Dhillon GS, Bleweft CJ, et al. A large adrenal teratoma in a neonate. Am Surg 2009;75:347-9.

17. Edwin M, Posadas ECK. Tumor angiogenesis and metastasis: Biology and clinical experience. In: Hoskins WJ, ed. Principles and Practice of Gynecologic Oncology. 4th ed. Baltimore, Md: Lippincott Williams \& Wilkins; 2005:73-91.

18. Gershenson DM, Del Junco G, Copeland $U$, et al. Mixed germ cell tumours of the ovary. Obstet Gynecol 1984;64:200-6.

19. Pectasides $D$, Aravantinos $G$, Visvikis $A$, et al. Platinum-based chemotherapy of primary extragonadal germ cell tumours: The Hellenic Cooperative Oncology Group experience. Oncology 1999;57:1-9. http:// dx.doi.org/10.1159/000011993

Correspondence: Dr. Dexin Yu, Department of Urology, The Second Hospital of Anhui Medical University, China; yudexin0718@sina.com.cn 